\title{
THE EVOLUTION OF QUASI-STELLAR SOURCES AND RADIO GALAXIES
}

\author{
M. RYLE \\ (Mullard Radio Astronomy Observatory, Cavendish Laboratory, Cambridge)
}

There seem to be two promising ways of investigating the evolution of radio sources: (a) the collection of data on a large number of sources so that attempts may be made to arrange them in an evolutionary sequence, (b) the study of a few sources, for which really detailed information is obtainable, to gain insight into the physical mechanisms occurring.

It is important to distinguish between the most powerful radio sources, the majority of which appear to have a simple double structure at the resolutions now attainable, and the more complex sources. The structure of the latter suggests that energetic particles have been accelerated in multiple 'events', each being of smaller magnitude than the events which give rise to the double sources; the total energy released may, however, be comparable.

It is evident that the evolution of the complex sources presents a more difficult problem, so it will be preferable to try to explain the double sources for a start. A simple model of these has been suggested (Ryle and Longair, 1967), which supposes that two clouds of relativistic plasma are ejected with equal velocities in opposite directions from the parent galaxy. From a consideration of the radiation lifetime and the physical dimensions of the more extensive sources it is evident that the speed of ejection must be comparable with the speed of light. The existence of such velocities implies that the two components will, in general, be observed at significantly different ages and they will have different angular separations from the galaxy. By examining a large number of sources it is then possible on the basis of this model to derive the power and age of each of the components. When the results are combined it appears that the model provides a reasonably consistent picture for the evolution not only of the extensive radio galaxies but also the QSS; the wide dispersion in the radio power and physical dimensions can be attributed mainly to the different ages at which the sources are observed.

From these results it appears that at high frequencies (where the effects of selfabsorption do not persist for long) the emission from each plasma cloud remains relatively constant for a period of about $10^{5}$ years despite the considerable increase in the size of the cloud which occurs during this time. At greater ages the further expansion is accompanied by a rapid decrease and the emission falls to a small value after about $3 \times 10^{6}$ years. The second phase corresponds to the expected variation of emis-

Perek (ed.), Highlights of Astronomy, 380-383. I.A.U. 
sion from a cloud which is expanding adiabatically, but the first phase seems to require a progressive enhancement of the magnetic field; there is good evidence (Williams, 1966) from the spectra of some of the most compact QSS (3C 147, 286 and 287) that the fields are initially small $\left(10^{-5}-10^{-4}\right.$ gauss). This model of powerful sources is therefore consistent with the sudden release of at least $10^{61}$ ergs in the form of relativistic particles in an initially weak magnetic field; it requires a mechanism for the amplification of this field as the plasma clouds move out through the galaxy.

Whilst such an analysis provides some evidence on the physical mechanisms involved, it is likely that further information will be obtained by studying sources which have a complex structure. A number of sources are known in which four main components are distributed along a line, suggesting that two separate periods of particle production have occurred (3C 46, 274·1, 452). In other cases (e.g. 3C 465), which have even more components, estimates may be made (Macdonald et al., 1966) of the variation of magnetic field with distance from the galaxy; it may then be possible to infer how the magnetic field in each component changes with time.

In some sources, such as the Seyfert galaxy NGC 1275, it seems that a more or less continuous acceleration of particles occurs. Early studies of the source (3C 84) revealed a compact component and an extensive halo some $30^{\prime}$ of arc in diameter. The work of Dent (1966) on the variability, of Pauliny-Toth and Kellermann (1966) on the spectrum, and of Barber et al. (1966) using long base-line interferometry have indicated that there are components having angular diameters $<0.001^{\prime \prime}$ of arc and $0.05^{\prime \prime}$ of arc. These components are coincident with the Seyfert nucleus.

Recent observations (Ryle and Windram, 1968) of this region with the 1-mile telescope at Cambridge enable 6 separate but associated components to be distinguished (Figure 1). In addition to those mentioned already there is one $5^{\prime}$ of arc in diameter, the same size as, and coincident with, the outer envelope of gaseous filaments of NGC 1275 . Another $(3 \mathrm{C} 83 \cdot 1 \mathrm{~B})$ is clearly associated with the neighbouring galaxy NGC 1265 and consists of an intense core with a curved tail extending away from NGC 1275. Yet another coincides with the $14^{\mathrm{m}}$ galaxy IC 310 , and this also shows an extension away from NGC 1275. The remarkable shape of these two additional sources confirms the suggestion by the Burbidges (1965) that the emission from NGC 1265 has its origin in NGC 1275.

We may now examine all the components in order to establish their energy requirements from their spectra, physical size, and radio power. In the case of the most compact component of $3 \mathrm{C} 84$, particles having a total minimum energy of $3 \times 10^{52}$ ergs are necessary if it is supposed that protons having 100 times the electron energy are present. The particle production has probably occurred within a period of less than 1 year and, as discussed by Van der Laan (1966) and Kellermann (1966), the lifetime of the source is likely to be limited to a few years by their escape from the region. The observed variability of this component is therefore to be expected, and the greater part of the electron energy will escape into the surrounding medium. 


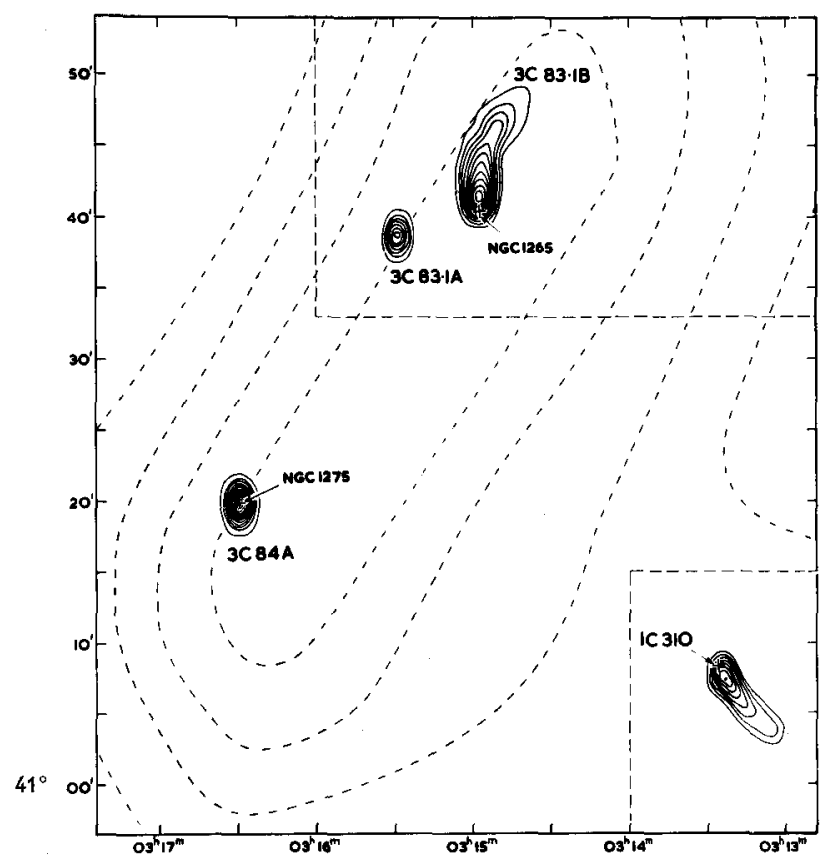

FIG. 1. Map obtained at $408 \mathrm{MHz}$ of the sources around NGC 1275.

The 0.05 of arc component, which is comparable in size with the Seyfert nucleus, requires a total particle energy of $\approx 10^{53}$ ergs; the electron life may be determined by a radiation loss time of $\approx 100$ years or by escape from the nucleus in a rather shorter time, but a supply of the order $7 \times 10^{51}$ ergs per year is necessary in either case. A similar rate is needed to account for the $5^{\prime}$ of arc component.

These results agree well with the conclusions of Dibaj and Pronik (1966) from optical studies. They find two components of the nucleus - one of which is about $0 \cdot 1$ parsec in diameter and has a gaseous mass of $35 M_{\odot}$, while the other has a diameter of 10 parsec and a gaseous mass of $10^{4} M_{\odot}$. These components are of size comparable with, and may be identified with, the two smallest radio-emitting regions. This supports the suggestion of Dibaj and Pronik that their origin can be attributed to supernova activity within the Seyfert nucleus since the particle energy associated with the most compact source is comparable with that in Cassiopeia A (J.E. Baldwin, private communication).

If we consider the sources associated with NGC 1265 and IC 310, it appears impossible on energetic grounds to explain the emission except on the basis of the interaction of relativistic particles ejected from NGC 1275 with the magnetic fields of these galaxies. The extensive halo component of 3C 84 may similarly be due to the interaction of particles with fields in the intergalactic medium. The total particle energy required is about $3 \times 10^{53}$ erg per year continuing for a period of about $5 \times 10^{6}$ years. 
Provided that the particles are trapped this conclusion is not dependent upon an assumption of equipartition of energy since a reduced rate of emission from each electron leads to a correspondingly longer electron life.

The results therefore suggest that NGC 1275 has been responsible for the continuous production of energetic particles over the past $8 \times 10^{6}$ years at a rate which has decreased from about $3 \times 10^{53}$ to $2 \times 10^{52}$ ergs per year. The total particle energy released is then at least $4 \times 10^{60} \mathrm{ergs}$ and is comparable with that of the most powerful QSS and radio galaxies known. NGC 1275 differs in that the energy release takes place over some millions of years rather than over $10^{2}-10^{3}$ years only. This difference of time-scale may be explicable in terms of energy production by supernova explosions. In the case of NGC 1275 the explosion of one supernova every 10 years could provide the energy to maintain all three radio components and could continue for several million years, whereas in the powerful sources a chain reaction of supernovae, as suggested by G.R. Burbidge (1961), might perhaps release the same total energy within 100 years, a period comparable with the light travel time across the nucleus.

It seems therefore that, besides the age of the source, the rate at which energy is released may be of great importance in determining its character. The intrinsically most powerful are likely to have a brief period of unusual optical emission and will have the simplest radio structure. An increase of the time during which particle acceleration occurs, from say $10^{2}$ to $10^{4}$ years, is unlikely to have much effect on the radio emission except at very high frequencies, but may result in a marked reduction in the optical emission. A further increase in this time may cause the optical peculiarities to be so faint as to be undetectable except for the nearest sources, while still producing intense radio emission. Alternatively, if a series of brief events of moderate power occur, spaced at long intervals, relatively intense optical sources might be produced without appreciable radio emission. Are these the radio quiet QSO's?

\section{References}

Barber, D., Donaldson, W., Miley, G. K., Smith, H. (1966) Nature, 209, 753.

Burbidge, G.R. (1961) Nature, 190, 1053.

Burbidge, E. M., Burbidge, G.R. (1965) Astrophys. J., 142, 1351.

Dent, W.A. (1966) Astrophys. J., 144, 843.

Dibaj, E.A., Pronik, V.I. (1966) Jzv. krym. astrofiz. Obs., 35, 87.

Kellermann, K.I. (1966) Astrophys. J., 146, 621.

Macdonald, G.H., Neville, A.C., Ryle, M. (1966) Nature, 211, 1241.

Pauliny-Toth, I. I. K., Kellermann, K.I. (1966) Astrophys. J., 146, 634.

Ryle, M., Longair, M.S. (1967) Mon. Not. R. astr. Soc., 136, 123.

Ryle, M., Windram, M.D. (1968) Mon. Not. R. astr. Soc., 138, 1.

Van der Laan, H. (1966) Nature, 211, 1131.

Williams, P.J.S. (1966) Nature, 210, 285. 